\title{
LAUR- $00-2162$
}

\section{INTEGRATION OF TGS AND CTEN ASSAYS USING THE CTEN_FIT \\ ANALYSIS AND DATABASING PROGRAM}

\author{
Robert J. Estep, Connie Buenafe, ${ }^{a}$ and Sheila Melton \\ Los Alamos National Laboratory \\ P.O. Box 1663 \\ Los Alamos NM 87545 \\ restep@lanl.gov, cbuenafe@lanl.gov,smelton@lanl.gov
}

\begin{abstract}
The CTEN_FIT program, written for Windows 9x/NT in $\mathrm{C}++$, performs databasing and analysis of combined thermal/epithermal neutron (CTEN) passive and active neutron assay data and integrates that with isotopics results and gamma-ray data from methods such as tomographic gamma scanning (TGS). The binary database is reflected in a companion Excel database that allows extensive customization via Visual Basic for Applications macros. Automated analysis options make the analysis of the data transparent to the assay system operator. Various record browsers and information displays simplify record keeping tasks.
\end{abstract}

\section{INTRODUCTION}

The recently developed CTEN_FIT 1.0 software, ${ }^{1}$ which has been licensed to Canberra Industries ${ }^{2}$ for distribution, was developed for analyzing passive and active neutron assays performed with the combined thermal/epithermal neutron (CTEN) ${ }^{3-5}$ prototype recently deployed at the Los Alamos RANT facility. A secondary goal was to simplify record-keeping by databasing the data files, methods, and results of every analysis and placing copies of all database records in a Microsoft Excel spreadsheet for easy access by the user. The connection with Excel via OLE (object linking and embedding) automation has the added advantage of allowing extensive customization of analysis methods and reporting through Visual Basic for Applications (VBA) macros, which are Visual Basic programs that have direct access to the contents of the Excel speadsheet. Using VBA macros, the user can can extract selective information from the database records; perform calculations; create dialogs; add additional information to each record as it is created; call user-written $\mathrm{C}++$ or Fortran modules; and in general add any kind of analysis algorithms or output forms desired.

\footnotetext{
${ }^{a}$ Under contract to Los Alamos National Laboratory from Allied Signal Corp.
} 


\section{DISCLAIMER}

This report was prepared as an account of work sponsored by an agency of the United States Government. Neither the United States Government nor any agency thereof, nor any of their employees, make any warranty, express or implied, or assumes any legal liability or responsibility for the accuracy, completeness, or usefulness of any information, apparatus, product, or process disclosed, or represents that its use would not infringe privately owned rights. Reference herein to any specific commercial product, process, or service by trade name, trademark, manufacturer, or otherwise does not necessarily constitute or imply its endorsement, recommendation, or favoring by the United States Government or any agency thereof. The views and opinions of authors expressed herein do not necessarily state or reflect those of the United States Government or any agency thereof. 


\section{DISCLAIMER}

Portions of this document may be illegible in electronic image products. Images are produced from the best available original document. 
The CTEN method, like other passive active neutron (PAN) assay methods, combines two analysis techniques: passive neutron coincidence counting (PNCC) and the active differential dieaway technique (DDT). Several options are available for analyzing this data. Depending on the analysis path, the two methods may be used separately or in combination. Some CTEN analysis options, for example, use active data to derive matrix corrections for use in the passive analysis. It was desired that CTEN_FIT offer the option to integrate the analysis of PNCC and DDT assays with isotopic composition information generated by programs such as $\mathrm{MGA}^{6}$ or FRAM, ${ }^{7}$ so that output forms summarizing analysis results could report masses for the isotopes required by WIPP. With all this complexity already required, we decided to go a step further and add support for the databasing and analysis of data from gamma-ray methods such as TGS (tomographic gamma scanning). Thus, CTEN_FIT provides for the simultaneous analysis and databasing of four different techniques at once: DDT, PNCC, isotopics, and TGS.

Specific data file types and formats are required for analysis of the CTEN_FIT DDT and PNCC method data, to support the significant "built-in" analysis capabilities for those methods. However, for the isotopics and TGS analyses no part of the analysis is built in, not even file reading. All that is provided is a base level of databasing (file names, etc.), the ability to specifiy analysis options in method records, and the ability to register VBA macros to be called for file reading, analysis, and output. The TGS support also allows interfacing to the Los Alamos TGS_FIT software, which is used for image reconstruction and analysis of TGS, DDT, and PNCC data. Because of this flexibility, referring to the fourth analysis method as TGS is merely a suggestion. Any kind of analysis of any kind of data can be incorporated via this fourth data type. As an example, we have plans to database and integrate non-TGS transmission-corrected gammaray assays of crate counters in the IBIS $^{8}$ system using the "TGS" support in CTEN_FIT.

Mastery of the CTEN_FIT program requires some effort, because of the databasing and the extensive setup options. However, once set up its use can be made completely transparent to the NDA operator through automatic analysis options. The recent application to the $\mathrm{CWAM}^{9}$ (crated waste assay monitor) instrument delivered to Oak Ridge/Y12 is a case in point. In the base configuration, the CWAM operator performs an active DDT assay (no passive assay is done, as the assay is for uranium) using the the Los Alamos WIN_CTEN ${ }^{10}$ data acquisition software. When the assay is finished, WIN_CTEN transmits the "c10_analyze_all" message to the CTEN_FIT program via the Windows message queue (i.e., using the Windows API PostMessage() function). This initiates the CTEN_FIT automatic database update and analysis 
sequence, which reads data files from a specified active data directory into the database and unen $\$$

analyzes any unanalyzed data records using a method specified for automatic analysis (up to eight different methods can be specified for automatic analyses, but CWAM currently uses only one). A custom VBA print macro is then called to print out results of the assay. All this happens in the background with no intervention required on the part of the operator.

Automatic analysis is provided to reduce the skill and training requirements for new operators, but a direct, foreground use of the CTEN_FIT software offers many powerful features that are worthwhile to learn. These include an electronic logbook that automatically records all events and user actions as well as database access features that allow browsing and manipulation of previous assays and analyses. Full documentation of these features can be found in the "CTEN_FIT User's Manual" (ref. 1) and related documentation. In this article we summarize some of the main features available.

\section{SPECIFICATIONS}

The CTEN_FIT version 1.0 program was written in $\mathrm{C}++$ using the Microsoft Visual $\mathrm{C}++6.0$ compiler. It runs under Windows 95 and 98 . Although it has never been tested with any versions of Windows NT, there is no reason to think it would not run under NT operating systems, as no special hardware interfacing is involved. We have tested CTEN_FIT on 133-MHz and faster PC clone computers and saw no performance problems. The slowest parts of the program are those that involve transfer of data between CTEN_FIT and Microsoft Excel via OLE, which involve noticable time delays. We are examining methods to speed that transfer in future revisions.

The currently distributed version (1.0) of CTEN_FIT runs only with Microsoft Office 97' installed, and not with the recent Microsoft Office 2000.

\section{THE CTEN_FIT DATABASE}

There are six primary record types in CTEN_FIT and several supporting record types. The four primary data record types have subtypes for normal, calibration, and background data and analyses. All the CTEN_FIT record types are listed in Table I, below. 
TABLE I. CTEN_FIT Database Record Types and Subtypes

\begin{tabular}{|c|c|c|c|}
\hline Type & Subtype & Excel Worksheet & Description \\
\hline Active & normal assay & Active Data & \multirow{3}{*}{$\begin{array}{l}\text { Active (DDT) data files are recorded, } \\
\text { along with some pre-analysis of scaler } \\
\text { mode data. }\end{array}$} \\
\hline Active & calibration & Active Calibration & \\
\hline Active & background & Active Background & \\
\hline Passive & normal assay & Passive Data & \multirow{3}{*}{$\begin{array}{l}\text { Passive (PNCC) data files are recorded. } \\
\text { No analysis is done. }\end{array}$} \\
\hline Passive & calibration & Passive Calibration & \\
\hline Passive & background & Passive Background & \\
\hline Isotopics & all & Isotopics Data & Isotopics results files are recorded. \\
\hline TGS & normal assay & TGS Data & \multirow{3}{*}{$\begin{array}{l}\text { TGS or other method file names are } \\
\text { recorded. }\end{array}$} \\
\hline TGS & calibration & TGS Calibration & \\
\hline TGS & background & TGS Background & \\
\hline Results & normal assay & Results & \multirow{3}{*}{$\begin{array}{l}\text { Results from an analysis are recorded, } \\
\text { with references to all data records and } \\
\text { the single method record employed. }\end{array}$} \\
\hline Results & calibration & Calibrations & \\
\hline Results & background & Background & \\
\hline Batch & - & Batch & For recording analysis batches. \\
\hline Method & - & Method & Description of an analysis method. \\
\hline \multicolumn{4}{|c|}{ The next two record types are referenced by method records. } \\
\hline $\begin{array}{l}\text { Neural } \\
\text { Network }\end{array}$ & - & Neural Network & $\begin{array}{l}\text { Description of a neural network or other } \\
\text { external executable to call. }\end{array}$ \\
\hline Tomography & - & Tomography & $\begin{array}{l}\text { Setup information for tomographic } \\
\text { imaging }\end{array}$ \\
\hline \multicolumn{4}{|c|}{ The remaining entries are not records, but spreadsheets that can be referenced } \\
\hline- & - & Constants & User's constants \\
\hline- & - & Setup & Copy of database setup file \\
\hline- & - & Scratch Pad & Used to communicate with VBA macros \\
\hline
\end{tabular}

The CTEN_FIT database is actually a binary file with default extension .CDB, which is mirrored (almost) in an Excel XLS file with the same title. Both are necessary. The Excel database file contains all user macros and, possibly, user-defined worksheets to support customized analysis, while the binary database contains indexing and security information not found in the Excel file. The Excel database contains a worksheet for each record type, with the names listed in Table I. The first row in each worksheet lists names for for the various information fields in each record. Starting in row two, each worksheet row contains a different record. Fig. 1 below illustrates a segment of the Excel database file for the Active Data worksheet, which stores Active Data records. The quantities listed in each column are scaler sums that are defined and named in the database setup file. These are used only in the CTEN system installed at RANT. The CWAM system uses a different set of scaler sums in its analysis.

.++++++++++ INSERT FIG. 1 
The user can create as many CTEN_FIT databases as desired, although only one database can be accessed at a time. It is a matter of taste whether to put a year's worth of data into every database or to start a new database every week.

The databasing in CTEN_FIT is intended to be used for the automation of quality assurance tasks that are now done by hand and to provide complete tracebility on all analyses. This is accomplished through various design and security features, which are too numerous to describe in detail here. We will mention just a few security features that make CTEN_FIT a useful quality assurance tool. Every record of any type is dated and given a unique serial number and can never be deleted from the database once entered. Method records, for example, can be freely edited without changing the method name, and will appear to the user to have been changed for subsequent analyses. However, the original record remains in the database and will still be associated with any analyses that referenced that particular version of the method. The creation date, last modification date, and size of external files used in analyses are monitored to ensure than no alteration or substitution of those files has occurred. There are (optional) separate passwords associated with the binary and Excel database files, preventing them from being opened by an unauthorized party either in CTEN_FIT or Excel. This feature also protects Excel user macros from being altered without authorization. The individual cells of the Excel database file can optionally be locked to prevent modification by any user (the randomly generated password, embedded in the binary CDB file, would be known only to the CTEN_FIT program). The automated electronic logbook, though not password protected itself, can be protected by user procedures and records all transactions that take place in the database.

All CTEN_FIT records contain generic information such as a unique record serial number, the time and date the record was created and who created it, and other bookkeeping information. Beyond that, the information stored varies by record type. The different record types ared described below.

\section{$\underline{\text { Data records }}$}

The data records (Active, Passive, Isotopics, and TGS) contain the file name and path of the data file(s) comprising a single analysis of that type, the name of the operator who collected the data and any comments, the sample ID, and similar information. The Active records use only WIN_CTEN-generated ADA, AQ1, and AQ2 data files (scaler mode data and shielded and bare 
detector list-mode files, respectively), and require those file extensions and formats. Assay data sufficient for most non-imaging, non-list-mode DDT analyses is extracted from the ADA scalermode files and stored in the Active record. The Passive records use only WIN_CTEN-generated PDA, PQ1, and PQ2 data files (scaler mode data and shielded and bare detector list-mode files, respectively). Bookkeeping information (operator name, comment, etc.) is extracted from the PDA file and stored in the Passive record, but no real data is stored. Bookkeeping information and data is read into Isotopics and $T G S$ records by user-written, custom VBA macros that are registered in the database setup file used to generate the database. The file types supported are also specified in the setup file by their file extensions. We currently use a macro named READ_FRAM and the TXT extension to read in FRAM ASCII output files, and a macro named $R E A D \_T M A$ and the TMA extension for preanalyzed TGS results files in our test/example cases.

\section{Method and Related Records}

Each Method record defines a way of analyzing the data by specifying the data types required for the analysis and the specific analysis methods to be used. Generally, CTEN_FIT only does part of a passive or active neutron analysis, leaving the remaining analysis and reporting of results for two Neural Network executables, three sets of Tomography matrix image reconstructions, and three user-defined VBA macros to carry out. The way this is done is illustrated in fig. 2, below, which shows the Edit Method Record dialog used for creating and editing Method records. This dialog is accessed by clicking the Setup/Method Setup menu option, followed by either the Edit Method or Add New Method buttons. The particular method shown, named "PAN with isotopics," uses the external executable defined in a Neural Network record named "PAN Matrix CF" to create a matrix correction factor for the active analysis (although it is not obvious, a matrix correction is also returned for the passive analysis). The built-in passive list mode analysis generates various histograms, moments, and coincidence rates used in the Gray Barrel analysis

methods of Brunson, et al.. ${ }^{11}$ The remaining active and passive analysis is done by VBA macro I, named $P A N \_M A C R O$, and by macro II, named PassOnly. The PAN_MACRO macro calls two other macros, ActMac and PassMac2, in succession, which is the method used to expand the three-macro-per-method allotment to an arbitrary number of macros. Preanalyzed isotopics results are integrated into the output in the print macro named PAN_ISO_Print, which reports one active and two passive results (from two different methods). A recommendation of which result to use is made, based on various indicators. The check box in the lower right flags this method for use in automatic analyses. 
At first glance the dialog in fig. 2 may seem complex, but most of the detail is encapsulated by simply named references to supporting records and macros, or by supporting dialogs. The automatic analysis and passive list options are set in the "Advanced" dialogs for those two categories. The Neural Net and Tomography selections correspond to records of those types, with their detailed settings encapsulated in a single name that displays in drop-down lists. The VBA macros can conceal an unlimited level of detail. Considering that it totally specifies an integrated analysis of up to four measurement techniques at once, or any combination therefore, it's actually a fairly simple dialog to use.

Figure 3 shows the Add Neural Network/Exe Record dialog box, accessed by clicking the Setup/Neural Network Setup menu option and then the Add Record button. This dialog is being used to create the Neural Network record named "PAN Matrix CF." The record specifies that at a well defined point in the analysis sequence an external executable file named NN.EXE will be spawned with the command line "pan $2 \times 2 . \mathrm{cmd}$." Information in the specified columns in the "input" Active Data worksheet is placed in a ASCII file named "temp.in" to be used as input by the executable (NN.EXE). The executable is expected to create an ASCII file with the name "temp.out," which will be read by CTEN_FIT to extract four floating point numbers that will be stored in the specified columns in the "output" Results worksheet. The rows to be accessed in the input and output worksheets correspond to those of the Active and Results records involved in the analysis. (The rule is that for the input worksheet, if a data-type worksheet is referenced and is involved in the analysis, the row number is determined by that record's index. In all other cases the Results index will be used to compute the row number.) Data and Results worksheets that have subtypes will be permuted to the correct subtype. That is, if the analysis is performed on background data, the Active Background and (results) Background worksheets will be accessed instead of the specified Active Data and Results worksheets, which are for normal assays.

In the Neural Network record being created in fig. 3, the NN.EXE program called is a probabilistic neural network program originally called PNN that was modified to allow a

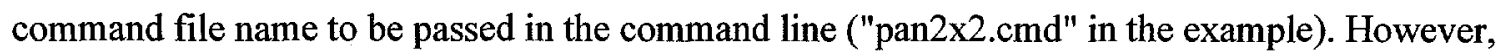
the executable does not have to be a neural network program, but can be any kind of program. Note that whenever a Neural Network record is created a test is done to verify the existence of the executable. Its creation date, last modification date, and size are recorded in the Neural Network record. If any of these change, an error will be generated whenever that Neural Network 
record is accessed in an analysis. This protects against alteration or substitution of the executable, which in effect would be an alteration of the method itself and would defeat the security measures that safeguard the integrity and tracebility of the Method and Results records.

The Tomography record stores setup information, such as scanning resolutions and other dimensions, along with the names of the binary tomographic matrix files (EMT, AMT, and TMT files) that are used in the TGS_FIT ${ }^{12,13}$ program to define the geometry of the problem. A command file name can also be specified that will perform the TGS_FIT analysis. As with Neural Network records, all files referenced by the Tomography record are safeguarded by verification of their creation and last modification dates and file size.

\section{Results Records and the Analysis Queue}

A Results record, which is also be called an "analysis set," records the details of every analysis, referencing back to the data records used in the analysis as well as to the Method record that describes how the analysis will be or was done. The ambiguity over whether the analysis was done or not exists because one initially creates "partial" Results records that include all the information needed for the analysis, but that have not been analyzed yet. These unanalyzed records are said to be in the "analysis queue" awaiting submission for analysis. One can create as many partial results records as desired before submitting them for analysis. Schematically, this can be represented as

$$
\begin{aligned}
\text { (As required: } \text { Active }+ \text { Passive } & + \text { Isotopics }+ \text { TGS) } \\
& + \text { Method } \rightarrow \text { partial Results (added to queue) }
\end{aligned}
$$

followed by processing of the analysis queue (for all partial Results records),

$$
\text { partial Results } \rightarrow \longrightarrow \text { completed Results records. }
$$

Results records can be created and processed automatically by pressing the Run Analyses button on the main window, which both creates the analysis queue and then submits it for analysis, or equivalently by clicking the Analysis/Update Analysis List and Analysis/Run Analysis List menu options in succession. These, as implied, create the analysis queue and submit it for 
analysis, respectively. The automatic analysis loops through all the Methods flagged as automatic and searches the database by subtype for data records that are required by the method and that have not yet been analyzed by that method. Following a set of simple rules based on the declared sample ID, data records of the different required types are associated into a partial Results record. Only data records with the same sample ID will be associated into a Results record and no data record will be used twice for the same method. Therefore, there can be left-over data records that could not be matched and would presumably be picked up in later automatic analyses.

Much greater flexibility is allowed in manually associating data files with a method to create a partial Results record. Figure 4 shows the Create Analysis Sets dialog used for this purpose, which is accessed by clicking the Analysis/Create Analysis Sets menu option. The "Pan with isotopics" method is selected as the Method record to be used, and data records of subtype "Normal" are listed by type in four list boxes. The data types that are required in this method (Active Data, Passive Data, and Isotopics, but not TGS Data) are indicated by check marks. In this example case the user has followed the recommended file naming convention of naming data files by their sample ID plus a ordinal number used to distinguish repeat assays. The WIN_CTEN software offers this convention as one method of automatic file naming (the others conventions offered are based on the date or a consecutive run number). The sample ID that appears on the first line in the small information box below each list box came from the data file itself, except in the case of the isotopics (.TXT) file, which does not store that information. The READ FRAM macro (macro II) registered for this database (independent of the method) uses the file name itself to deduce the sample ID and enter it in the database. To create a results record, one selects one of each required data type and presses the Add to Queue button, which creates a partial results record and displays it as a single line-entry in the information box at the bottom of the dialog.

In the example in fig. 4, three previously created partial results records are shown (the entries show the results record serial number, the method name, then the file names for the data records used in parentheses). These three partial results records constitute the normal portion of the current analysis queue (there could be additional, unshown entries for the calibration or background subtypes). Notice the results record number 848 , for which (presumably) no isotopics assay was done on the drum and the substitute assay "Air-13.txt" was used instead. This sort of substitution can only be done using the Create Analysis Sets dialog. Three data files with sample ID "56110" are currently selected and would be appended to the analysis queue if the Add to Queue button were clicked. 


\section{THE CTEN_FIT MAIN WINDOW}

Figure 5 shows the CTEN_FIT main window, with the Active Data record browser selected. The different browsers and information windows are selected by a combination of tab controls (along the top of the information window) and by the five buttons that run from left to right along the bottom, each of which brings up a different tab set. The Data, Background, and Calibration button switch between the browser displays for those subtypes, all of which have the same tab selections. The Images button brings up various tomographic image displays, ${ }^{b}$ while the History button brings up a scrolling edit box that shows the most recent automatic electronic logbook entries.

All necessary database functions (which excludes the browsers and other information displays) can be executed using the pull-down menu items. However, to simplify the most common operations, three useful buttons are located along the right side of the main window. The Update Database button reads new data files into the database, while the Run Analyses button commences automatic analysis. The Logbook button brings up a copy of the electronic logbook in an easy-to-use ASCII text editor (PFE32 ${ }^{14}$ ) that allows viewing, editting, and printing of the logbook. An excerpt from a typical logbook is shown below (some text to the right has been truncated).

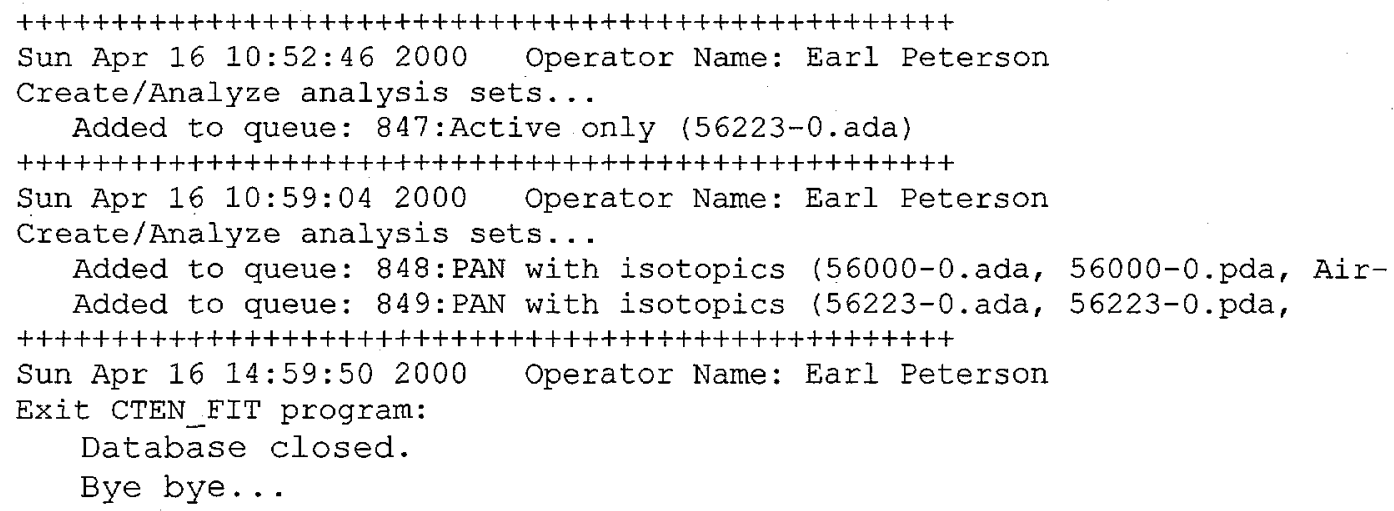

\section{CURRENT STATUS AND FUTURE WORK}

\footnotetext{
${ }^{b}$ The image displays are not implemented in the CTEN_FIT version currently in use in the field.
} 
A few important features, along with several minor ones, remain to be implemented in CTEN_FIT program. The most significant remaining work on version 1.x is to add full support for tomographic image reconstruction analysis techniques and graphical displays, which are currently implemented only in experimental versions of the code. This work is underway, and should be completed sometime in the year 2000 .

\section{NOMENCLATURE}

The following conventions are used in referencing various components of the CTEN_FIT software.

- Record types are indicated in bold italics (e.g., Active Background) and will have the same name as the label on their corresponding Excel worksheet. Data types with unspecified subtypes will be referenced the same way (e.g., Active), although there is no corresponding Excel worksheet in that case.

- Record subtypes (normal, background, and calibration) are written in ordinary type.

- Menu items are referenced in bold type, with a slash separating different levels of the menu (e.g., Analysis/Create Analysis Sets). Underscores and ellipses are suppressed. Dialog buttons or other controls are also referenced in bold tpye.

- Dialog names are referenced in underlined bold text (e.g., Create Analysis Sets)

- VBA macros are referenced in italics (e.g., READ_FRAM).

\section{ACKNOWLEDGEMENT}

This research was funded by the Mixed Waste Focus Area, Idaho National Engineering and Environmental Laboratory, and by the DOE, Environmental Management Science and Technology (EM-50).

\section{REFERENCES}

1. R. Estep, C. Buenafe, and S. Melton, "The CTEN_FIT User's Manual," Los Alamos Report NIS6-QAP-0018 (in revision) (April, 2000).

2. Canberra Industries, A Division of Packard BioSciences, Inc., 800 Research Parkway, Meridan Connecticut. 
3. S. Melton, R. Estep, C. Hollas, G. Arnone, G. Brunson, and K. Coop, "Development of Advanced Matrix Correction Techniques for Active Interrogation of Waste Drums Using the CTEN Instrument," Proc. INMM 38th Annual Meeting, Phoenix, AZ, July 20-24, 1997.

4. R. J. Estep, K. L. Coop, C. Hollas, S. Melton, and D. Miko, "Lump correction and identification in the combined thermal/epithermal neutron (CTEN) method", Proc. INMM 39th Annual Meeting, Naples Florida, July 26-30, 1998.

5. C. L. Hollas, et al., "Matrix Effects Corrections in DDT Assays of ${ }^{239} \mathrm{Pu}$ with the CTEN instrument," 5th Annual NDA and NDE waste characterization conference, Salt Lake City (January, 1997).

6. The MGA code is distributed by Canberra Industries. (see ref. 2)

7. The FRAM isotopics code is distributed by EG\&G Ortec, 100 Midland Road, Oak Ridge, Tennessee.

8. D. B. Syme, et al., "'IBIS' - the integrated box integration system", to be presented at this conference.

9. S. Melton, R. Estep, and E. Peterson, "Calibration of the crated waste assay monitor (CWAM) for the low-level waste measurements for the Y-12 plant," to be presented at this conference.

10. R. Estep, et al., "User's Manual for WIN_CTEN 1.0," NIS6-QAP-0024 (in revision) (April, 2000).

11. G. Brunson and N.J. Nicholas, "Shift-Register Neutron-Coincidence Counting and the Gray Barrel Problem," Los Alamos Rept. LA-12414-MS (Oct. 1992).

12. R. Estep, "TGS_FIT 3.1 User's Manual," to be published.

13. R. Estep and J. Cavender, "The WIN_TGS software package for tomographic gamma scanner systems," Proc. 35th INMM meeting, Naples, FL,(July 17-20, 1994).

14. The PFE32 software was written by Alan Phillips, Lancaster University Computer Center. Copies can be obtained from the web site www.winsite.com or www.simtel.net/simtel.net. 


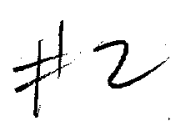

\begin{tabular}{|c|c|c|c|c|c|c|c|c|c|c|}
\hline & A & $\mathrm{AB}$ & ACW & AD & $\mathrm{AE}$ & AF & AG & AH & Al & $f$ \\
\hline 1 & efmTotal & $H$ & SfmSum & $+1-$ & Bfm4 & +1 & Dfm4 & +1 & BfmSum/Efm & \\
\hline 2 & 23412 & 153.01 & 33587 & 183.3 & 117270 & 342 & 30728 & 175.3 & 17.00012779 & \\
\hline 3 & 23507 & 153.32 & 34156 & 184.8 & 117871 & 343 & 31176 & 176.6 & 17.02646065 & \\
\hline 4 & 23349 & 152.8 & 33819 & 183.9 & 117036 & 342 & 31113 & 176.4 & 17.05807495 & \\
\hline 5 & 24669 & 157.06 & 35599 & 188.7 & 123017 & 351 & 32539 & 180.4 & 16.95597649 & \\
\hline 6 & 23615 & 153.67 & 34844 & 186.7 & 120374 & 347 & 31921 & 178.7 & 17.4002533 & \\
\hline 7 & 24864 & 157.68 & 36273 & 190.5 & 125365 & 354 & 33258 & 182.4 & 17.10702133 & \\
\hline 8 & 23822 & 154.34 & 35091 & 187.3 & 120983 & 348 & 31690 & 178 & 17.18902779 & \\
\hline 9 & 22543 & 150.14 & 9537 & 97.66 & 187312 & 433 & 36712 & 191.6 & 20.87543869 & \\
\hline 11 & 22433 & 149.78 & 9292 & 96.4 & 187624 & 433 & 36780 & 191.8 & 20.97820091 & \\
\hline 111 & 22910 & 151.36 & 9641 & 98.19 & 189388 & 435 & 36973 & 192.3 & 20.72143173 & \\
\hline 15 & วग5аח & 1 & ด्ग? & DE AF & 1АБАวค & 171 & 35115 & 100 & $2 \cap 71 \cap A 011 R$ & \\
\hline
\end{tabular}

Figure 1. A section of the Active Data worksheet from the Excel database portion of a sample database. Each row after the first corresponds to a separate Active Data record. The quantities shown are various scalers sums, which are named and defined in the database setup file. 


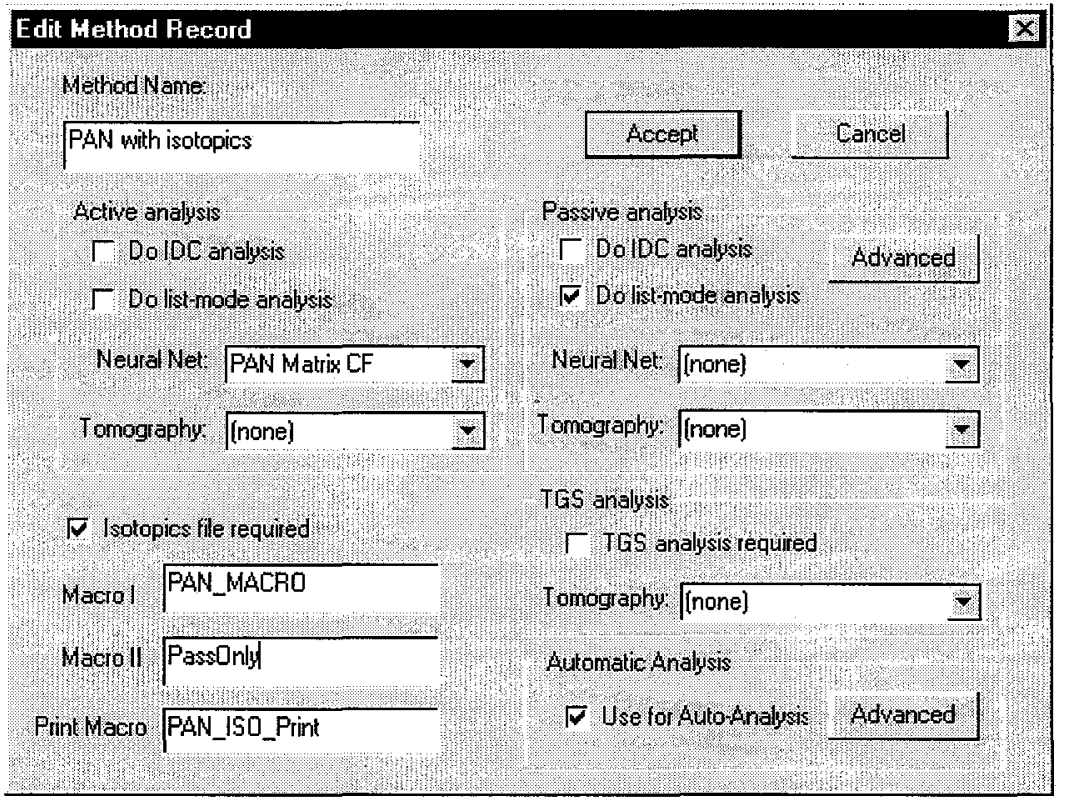

Figure 2. The Edit Method Record dialog, used for creating or editting method records.This dialog is accessed by clicking the Setup/Method Setup menu option, followed by the Add New Method or Edit Method buttons. 


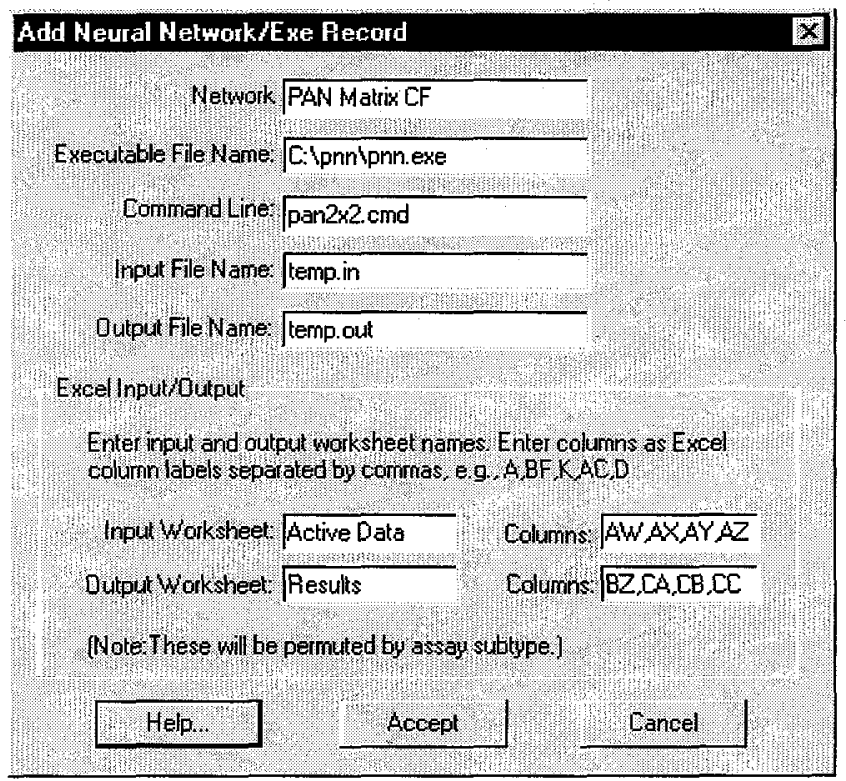

Figure 3. The Add Neural Network/Exe Record dialog used to create Neural Network records. This dialog is accessed by clicking the Setup/Neural Network Setup menu option, followed by the Add Record button. 


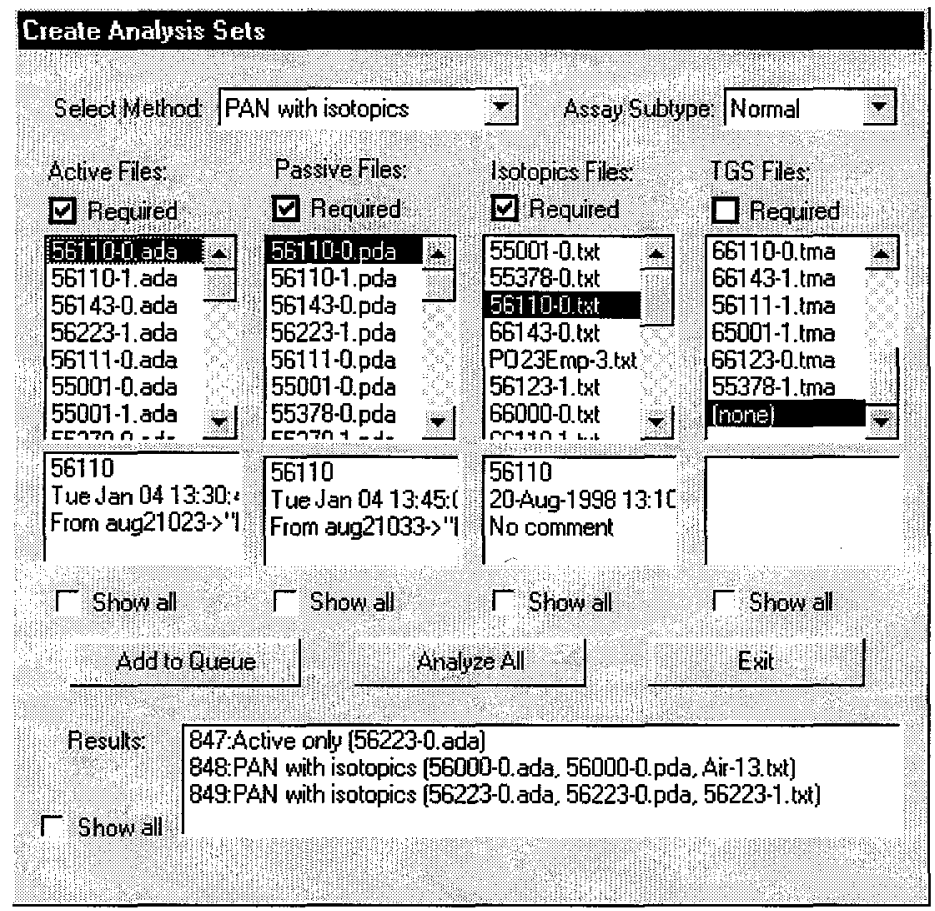

Figure 4. The Create Analysis Sets dialog, used to associate data records with a Method record to create a Results record in the analysis queue. This dialog is accessed by clicking the Analysis/Create Analysis Sets menu option. 


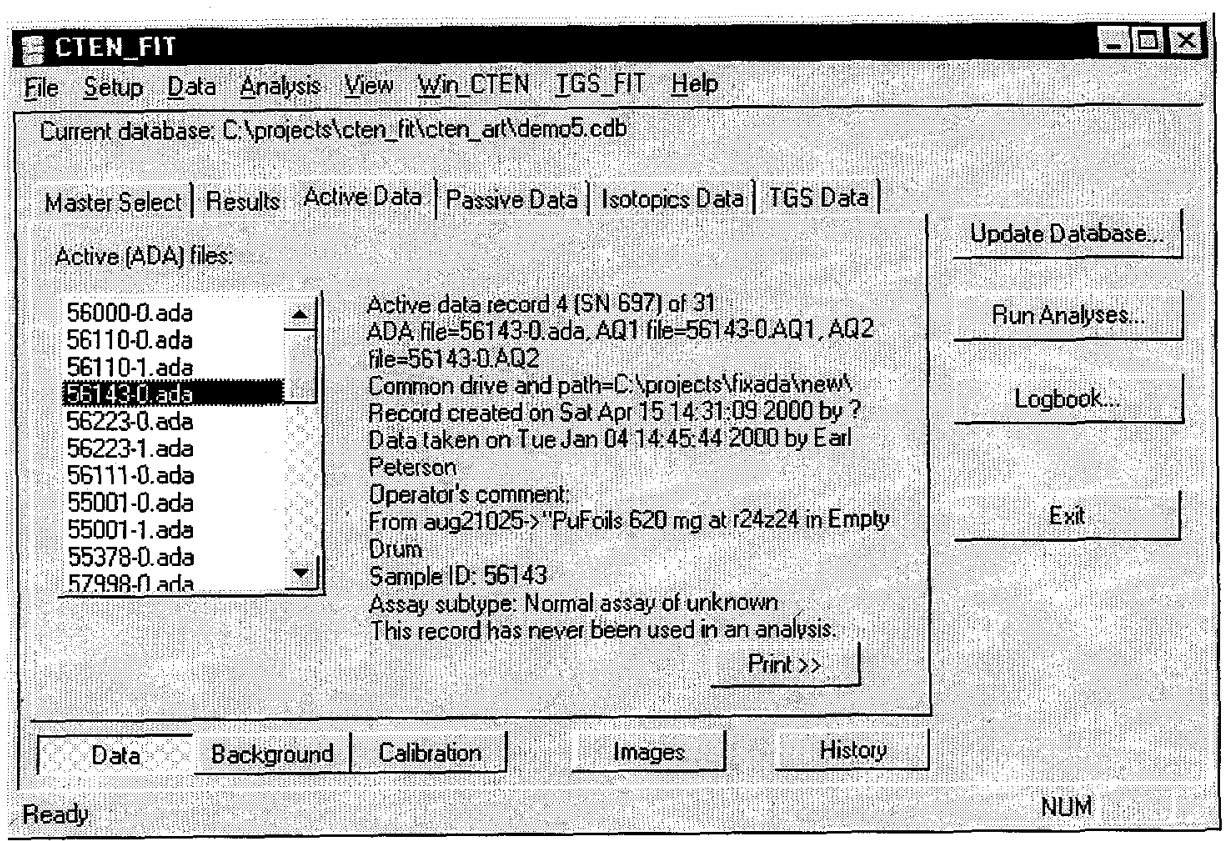

Figure 5. The CTEN_FIT main window, with the active (normal data) browser selected. Clicking the Print button will print detailed information about the selected record to the default printer. 